MKPH-T-03-2

\title{
Renormalization of relativistic baryon chiral perturbation theory and power counting
}

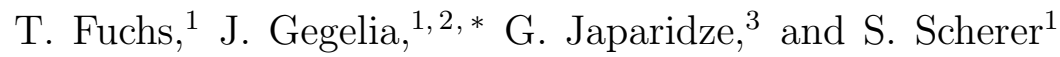 \\ ${ }^{1}$ Institut für Kernphysik, Johannes Gutenberg-Universität, D-55099 Mainz, Germany \\ ${ }^{2}$ High Energy Physics Institute, Tbilisi State University, \\ University St. 9, 380086 Tbilisi, Georgia \\ ${ }^{3}$ Center for Theoretical Studies of Physical Systems, \\ Clark Atlanta University, Atlanta, Georgia 30314, USA
}

(Dated: July 1, 2003)

\begin{abstract}
We discuss a renormalization scheme for relativistic baryon chiral perturbation theory which provides a simple and consistent power counting for renormalized diagrams. The method involves finite subtractions of dimensionally regularized diagrams beyond the standard $\overline{\mathrm{MS}}$ scheme of chiral perturbation theory to remove contributions violating the power counting. This is achieved by a suitable renormalization of the parameters of the most general effective Lagrangian. In addition to its simplicity our method has the benefit that it can be easily applied to multiloop diagrams. As an application we discuss the mass of the nucleon and compare the result with the expression of the infrared regularization of Becher and Leutwyler.
\end{abstract}

PACS numbers: 11.10.Gh,12.39.Fe

*Alexander von Humboldt Research Fellow 


\section{INTRODUCTION}

Starting from the pioneering work of Weinberg in 1979 [1], effective field theory has evolved into one of the most important theoretical tools for investigating strong-interaction processes in the low-energy regime. The concept of spontaneous symmetry breakdown, leading to the appearence of massless Goldstone bosons with vanishing interactions in the zero-energy limit, was already well-known in the beginning of the 1960s [2, 3, 4, 5]. Explicit symmetry breaking was taken into account in the framework of current algebra in combination with the partially conserved axial-vector current (PCAC) hypothesis [6] (for an overview see, e.g., 7, 8, 9]). Already in the 1960s, Weinberg realized that the predictions derived from current algebra could be reproduced in the framework of the so-called phenomenological approximation (tree-level diagrams) of an effective Lagrangian [10]. The key progress due to Weinberg's approach in 1979 was to systematically analyze corrections to the leading soft-pion results invoking a perturbative scheme not in terms of a coupling constant but rather in terms of external momenta and the pion mass [1]. Because of spontaneous symmetry breaking such an expansion is expected to work for momenta which are small compared to some intrinsic scale of the underlying theory. Since the starting point is a nonrenormalizable theory, infinities encountered in the calculation of loop diagrams need to be removed by a renormalization of the infinite number of free parameters of the most general effective Lagrangian. However, as long as one includes all of the infinite number of interactions allowed by symmetries, from the point of view of removing divergences there is no difference between the so-called nonrenormalizable theories and renormalizable the-

ories 11. As will be discussed later in detail, the freedom of choosing a renormalization scheme [12] can be advantageously used to formulate a power counting for the perturbative calculation of renormalized diagrams.

The ideas of Weinberg were further developed and comprehensively applied by Gasser and Leutwyler [13, 14] in terms of the generating functional of color-neutral quark bilinears which, at low energies, is dominated by the exchange and interaction of Goldstone bosons. A correspondence between the loop expansion and the chiral expansion in terms of momenta and quark masses at a fixed ratio was set up. Chiral perturbation theory (ChPT) in the mesonic sector has generated a host of successful applications up to and including the two-loop level (for a recent review see, e.g., Ref. [15]). The extension to processes involving one external nucleon was developed by Gasser, Sainio, and Švarc [16]. One of the findings in their approach was that higher-loop diagrams can contribute to terms as low as $\mathcal{O}\left(q^{2}\right)$, where $q$ generically denotes a small expansion parameter such as, e.g., the pion mass. This "mismatch" between the chiral and the loop expansion has widely been interpreted as the absence of a systematic power counting in the relativistic formulation. Gasser, Sainio, and Švarc pointed out that the appearance of another scale, namely, the mass of the nucleon (which does not vanish in the chiral limit) is one of the origins for the complications in the baryonic sector. The heavy-baryon formulation of ChPT [17, 18] provides a power counting scheme which is very similar to the mesonic sector. The basic idea consists in expressing the relativistic nucleon field in terms of a velocity-dependent field, thus dividing nucleon momenta into a large piece close to on-shell kinematics and a soft residual contribution. Most of the calculations in the one-baryon sector have been performed in this framework (for an overview see, e.g., Ref. [19]) which essentially corresponds to a simultaneous expansion of matrix elements in $1 / m_{N}$ and $1 /\left(4 \pi F_{\pi}\right)$. Although this scheme leads to a straightforward power counting, its disadvantage is that, in some cases, it does not provide the correct 
analytic behavior even in the threshold regime [20]. Several methods have been suggested to reconcile power counting with the constraints of analyticity in the relativistic approach 21, 22, 23, 24, 25, 26, 27]. The most widely used technique is the so-called infrared regularization of Becher and Leutwyler 23] which has been applied in various calculations of baryon properties [28, 29, 30, 31, 32], pion-nucleon scattering 33, 34, mesonic U(3) chiral perturbation theory [35, 36, 37], a discussion of the generalized Gerasimov-Drell-Hearn sum rule and the spin structure of the nucleon [38, 39], and the ground-state energy of pionic hydrogen [40].

The purpose of this work is to devise a new renormalization scheme leading to a simple and consistent power counting for the renormalized diagrams of a relativistic approach. The basic idea consists in performing additional subtractions of dimensionally regularized diagrams beyond the modified minimal subtraction scheme employed in Ref. 16]. Our approach is motivated by an observation made in the context of nonrelativistic nucleonnucleon scattering, where the application of the minimal subtraction scheme proved to be problematic. It was shown that the use of an appropriately chosen renormalization condition allows one to solve the problem of an "unnaturally" large scattering length and to obtain a consistent power counting in the two-nucleon sector [41, 42, 43]. Essentially the same idea of using a suitable renormalization condition has been discussed in Refs. [24, 25] for a simplified model of the one-nucleon sector of relativistic baryon chiral perturbation theory. One of the advantages of this approach, besides its simplicity, is that it may also be easily used in the renormalization of higher-order loop diagrams.

Our work is organized as follows. In Sec. II we provide those elements of the most general effective Lagrangian which are relevant for the calculation of the nucleon self-energy. In Sec. III we illustrate our method by means of a simple dimensionally regularized one-loop integral and compare the result with the infrared regularization of Becher and Leutwyler. In Sec. IV] we apply our renormalization scheme to the calculation of the nucleon mass. General conclusions are presented in Sec. D

\section{THE EFFECTIVE LAGRANGIAN}

In this section we will briefly discuss those elements of the most general effective Lagrangian in the single-nucleon sector which are relevant for the subsequent calculation of the nucleon self-energy. The effective Lagrangian consists of the sum of the purely mesonic and the $\pi N$ Lagrangians, respectively,

$$
\mathcal{L}_{\text {eff }}=\mathcal{L}_{\pi}+\mathcal{L}_{\pi N}
$$

both of which are organized in a (chiral) derivative and quark-mass expansion 11, 13, 14, 16, 44, 45, 46, 47, 48],

$$
\begin{aligned}
\mathcal{L}_{\pi} & =\mathcal{L}_{2}+\mathcal{L}_{4}+\mathcal{L}_{6}+\cdots, \\
\mathcal{L}_{\pi N} & =\mathcal{L}_{\pi N}^{(1)}+\mathcal{L}_{\pi N}^{(2)}+\mathcal{L}_{\pi N}^{(3)}+\mathcal{L}_{\pi N}^{(4)}+\cdots,
\end{aligned}
$$

where the subscripts (superscripts) in $\mathcal{L}_{\pi}\left(\mathcal{L}_{\pi N}\right)$ refer to the order in the expansion. Counting the quark-mass term as $\mathcal{O}\left(q^{2}\right)$ [13, 49], the mesonic Lagrangian contains only even powers, whereas the baryonic Lagrangian involves both even and odd powers due to the additional spin degree of freedom. 
From the mesonic sector we only need the lowest-order Lagrangian $\left[\mathcal{O}\left(q^{2}\right)\right][13]$,

$$
\mathcal{L}_{2}=\frac{F^{2}}{4} \operatorname{Tr}\left(\partial_{\mu} U \partial^{\mu} U^{\dagger}\right)+\frac{F^{2} M^{2}}{4} \operatorname{Tr}\left(U^{\dagger}+U\right)
$$

where $U$ is a unimodular unitary $(2 \times 2)$ matrix containing the Goldstone boson fields. In Eq. (11), $F$ denotes the pion-decay constant in the chiral limit: $F_{\pi}=F[1+\mathcal{O}(\hat{m})]=92.4 \mathrm{MeV}$. Here, we work in the isospin-symmetric limit $m_{u}=m_{d}=\hat{m}$, and the lowest-order expression for the squared pion mass is $M^{2}=2 B \hat{m}$, where $B$ is related to the quark condensate $\langle\bar{q} q\rangle_{0}$ in the chiral limit [13].

In order to discuss the $\pi N$ Lagrangian, let

$$
\Psi=\left(\begin{array}{l}
p \\
n
\end{array}\right)
$$

denote the nucleon field with two four-component Dirac fields $p$ and $n$ describing the proton and neutron, respectively. The most general $\pi N$ Lagrangian $\mathcal{L}_{\pi N}$ is bilinear in $\bar{\Psi}(x)$ and $\Psi(x)$ and involves the quantities $u, u_{\mu}, \Gamma_{\mu}$, and $\chi_{ \pm}$(and their derivatives), which, in the absence of external fields, read

$$
u^{2}=U, \quad u_{\mu}=i u^{\dagger} \partial_{\mu} U u^{\dagger}, \quad \Gamma_{\mu}=\frac{1}{2}\left[u^{\dagger}, \partial_{\mu} u\right], \quad \chi_{ \pm}=M^{2}\left(U^{\dagger} \pm U\right) .
$$

In terms of these building blocks the lowest-order Lagrangian reads [16]

$$
\mathcal{L}_{\pi N}^{(1)}=\bar{\Psi}\left(i \gamma_{\mu} D^{\mu}-m+\frac{1}{2} \stackrel{\circ}{g}_{A} \gamma_{\mu} \gamma_{5} u^{\mu}\right) \Psi
$$

where $D_{\mu} \Psi=\left(\partial_{\mu}+\Gamma_{\mu}\right) \Psi$ denotes the covariant derivative (in the absence of external vector and axial-vector fields) and $m$ and $\stackrel{\circ}{A}_{A}$ refer to the chiral limit of the physical nucleon mass and the axial-vector coupling constant, respectively. We have not displayed the corresponding counterterms in $\mathcal{L}_{\pi N}^{(1)}$ which are understood to be fixed in such a manner that the pole position of the nucleon propagator as well as the axial-vector coupling constant (in the chiral limit) are not affected by loop contributions. The explicit expressions of these counterterms in lowest order were identified in Ref. [16].

For our purposes, we only need to consider four of the seven structures of the Lagrangian at $\mathcal{O}\left(q^{2}\right)$ [16, 46],

$$
\begin{aligned}
\mathcal{L}_{\pi N}^{(2)}= & c_{1} \operatorname{Tr}\left(\chi_{+}\right) \bar{\Psi} \Psi-\frac{c_{2}}{4 m^{2}} \operatorname{Tr}\left(u_{\mu} u_{\nu}\right)\left(\bar{\Psi} D^{\mu} D^{\nu} \Psi+\text { H.c. }\right) \\
& +\frac{c_{3}}{2} \operatorname{Tr}\left(u^{\mu} u_{\mu}\right) \bar{\Psi} \Psi-\frac{c_{4}}{4} \bar{\Psi} \gamma^{\mu} \gamma^{\nu}\left[u_{\mu}, u_{\nu}\right] \Psi+\cdots
\end{aligned}
$$

where H.c. refers to the Hermitian conjugate. While the Lagrangian $\mathcal{L}_{\pi N}^{(3)}$ does not contribute to the nucleon mass, at $\mathcal{O}\left(q^{4}\right)$ we need to consider the term

$$
-\frac{\alpha}{2} M^{4} \bar{\Psi} \Psi
$$

resulting in the contribution $\alpha M^{4} / 2$ to the nucleon mass. This term results from identifying the relevant part of the most general chiral Lagrangian at $\mathcal{O}\left(q^{4}\right)$. To be specific, the coefficient $\alpha$ of Eq. (44) is related to the parameters $e_{i}$ of the Lagrangian $\mathcal{L}_{\pi N}^{(4)}$ of Ref. [46] by

$$
\alpha=-4\left(8 e_{38}+e_{115}+e_{116}\right) .
$$




\section{EXTENDED ON-MASS-SHELL RENORMALIZATION VERSUS THE IN- FRARED REGULARIZATION OF BECHER AND LEUTWYLER}

The basic idea of our renormalization scheme consists of providing a rule determining which terms of a given diagram should be subtracted in order to satisfy a "naive" power counting by which one associates a well-defined power with the diagram in question. The terms to be subtracted are polynomials in small variables and parameters (external momenta and squared pion mass) and can thus be realized by a suitable adjustment of the counterterms of the most general effective Lagrangian. In other words, our proposition is to perform additional subtractions (finite in number) of dimensionally regularized diagrams beyond the modified minimal subtraction scheme employed in Ref. [16].

In order to illustrate our method and to compare it with the approach of Becher and Leutwyler [23], we will first consider as an example the dimensionally regularized one-loop integral

$$
H\left(p^{2}, m^{2}, M^{2} ; n\right) \equiv-i \int \frac{d^{n} k}{(2 \pi)^{n}} \frac{1}{\left[(p-k)^{2}-m^{2}+i 0^{+}\right]\left[k^{2}-M^{2}+i 0^{+}\right]},
$$

where $n$ denotes the number of space-time dimensions. The masses $m$ and $M$ refer to the (lowest-order) nucleon and pion masses, respectively. Such a type of integral is needed in, e.g., the calculation of the one-pion-loop contribution to the nucleon self-energy [16]. Dimensional regularization provides a convenient tool to handle the ultraviolet divergence resulting from the region where all components of $k^{\mu}$ get large. However, as it stands, the loop integral of Eq. (6) does not yet satisfy a simple chiral power counting. Using Eq. (6) we will propose a renormalization procedure generating a power counting for tree-level and loop diagrams of the relativistic effective field theory (REFT) which is analogous to that given in Ref. [50] (for nonrelativistic nucleons). As will be explained below, by subtracting a suitable number of counterterms in the integrand, ${ }^{1}$ we apply a renormalization scheme resulting in an effective cutoff $Q$ for loop integrals which is of the order of some small expansion parameters such as $Q^{2} \approx M^{2}$ and $Q m \approx\left|p^{2}-m^{2}\right|$ for the specific case of Eq. (6). Using the forest formula of Zimmermann [12, 51] allows one to systematically deal with any diagram. The relevant subtractions can be implemented by adjusting the coefficients of the most general effective Lagrangian, i.e., the corresponding counterterms are local (polynomial) in momentum [12], which implies that only a finite number of counterterms are needed for the subtraction of a specific diagram. In general, this will then allow us to apply the following power counting: a loop integration in $n$ dimensions counts as $Q^{n}$, pion and fermion propagators count as $Q^{-2}$ and $Q^{-1}$, respectively, vertices derived from $\mathcal{L}_{2 k}$ and $\mathcal{L}_{\pi N}^{(k)}$ count as $Q^{2 k}$ and $Q^{k}$, respectively. In total this yields for the power $D$ of a diagram in the one-nucleon sector the standard formula [50, 52 ]

$$
D=n N_{L}-2 I_{\pi}-I_{N}+\sum_{k=1}^{\infty} 2 k N_{2 k}^{\pi}+\sum_{k=1}^{\infty} k N_{k}^{N},
$$

where $N_{L}$ is the number of independent loop momenta, $I_{\pi}$ the number of internal pion lines, $I_{N}$ the number of internal nucleon lines, $N_{2 k}^{\pi}$ the number of vertices originating from $\mathcal{L}_{2 k}$, and $N_{k}^{N}$ the number of vertices originating from $\mathcal{L}_{\pi N}^{(k)}$. In the language of chiral perturbation

\footnotetext{
${ }^{1}$ Here we make use of the fact that we may take more subtractions than would actually be necessary for the sole purpose of enforcing (ultraviolet) convergence.
} 
theory, $Q$ counts as a small momentum, i.e., as $\mathcal{O}(q)$, with the net result that Eq. (6) , after renormalization, is expected to be of order $\mathcal{O}\left(q^{n-3}\right)$.

Let us turn to the discussion of Eq. (6). We make use of the Feynman parametrization

$$
\frac{1}{a b}=\int_{0}^{1} \frac{d z}{[a z+b(1-z)]^{2}}
$$

with $a=(p-k)^{2}-m^{2}+i 0^{+}$and $b=k^{2}-M^{2}+i 0^{+}$, interchange the order of integrations, and perform the shift $k \rightarrow k+z p$ to obtain

$$
H\left(p^{2}, m^{2}, M^{2} ; n\right)=-i \int_{0}^{1} d z \int \frac{d^{n} k}{(2 \pi)^{n}} \frac{1}{\left[k^{2}+p^{2} z(1-z)-m^{2} z-M^{2}(1-z)+i 0^{+}\right]^{2}} .
$$

Making use of

$$
\int \frac{d^{n} k}{(2 \pi)^{n}} \frac{\left(k^{2}\right)^{p}}{\left(k^{2}-A\right)^{q}}=\frac{i(-)^{p-q} \Gamma\left(p+\frac{n}{2}\right) \Gamma\left(q-p-\frac{n}{2}\right)}{(4 \pi)^{\frac{n}{2}}} \frac{A^{p+\frac{n}{2}-q},}{\Gamma\left(\frac{n}{2}\right) \Gamma(q)}
$$

with $p=0$ and $q=2$, we find

$$
H\left(p^{2}, m^{2}, M^{2} ; n\right)=\frac{1}{(4 \pi)^{\frac{n}{2}}} \Gamma\left(2-\frac{n}{2}\right) \int_{0}^{1} d z[A(z)]^{\frac{n}{2}-2},
$$

where

$$
A(z)=-p^{2}(1-z) z+m^{2}(1-z)+M^{2} z-i 0^{+}
$$

\section{A. Chiral limit}

For the sake of simplicity, let us for the moment restrict ourselves to the (chiral) limit $M^{2}=0$ and introduce

$$
C(z, \Delta)=z^{2}-\Delta z(1-z)-i 0^{+}, \quad \Delta=\frac{p^{2}-m^{2}}{m^{2}}
$$

so that we obtain

$$
H\left(p^{2}, m^{2}, 0 ; n\right)=\kappa(m ; n) \int_{0}^{1} d z[C(z, \Delta)]^{\frac{n}{2}-2}
$$

where

$$
\kappa(m ; n)=\frac{\Gamma\left(2-\frac{n}{2}\right)}{(4 \pi)^{\frac{n}{2}}} m^{n-4} .
$$

For the purpose of evaluating the integral of Eq. (11) we write ${ }^{2}$

$$
\int_{0}^{1} d z[C(z, \Delta)]^{\frac{n}{2}-2}=(-\Delta)^{\frac{n}{2}-2} \int_{0}^{1} d z z^{\frac{n}{2}-2}\left(1-\frac{1+\Delta}{\Delta} z\right)^{\frac{n}{2}-2}
$$

\footnotetext{
${ }^{2}$ The boundary condition is properly taken into account by replacing $m^{2} \rightarrow m^{2}-i 0^{+}$in the final expression.
} 
and apply Eqs. 15.3.1 and 15.3.4 of Ref. [53] to obtain

$$
H\left(p^{2}, m^{2}, 0 ; n\right)=\kappa(m ; n) \frac{\Gamma\left(\frac{n}{2}-1\right)}{\Gamma\left(\frac{n}{2}\right)} F\left(1,2-\frac{n}{2} ; \frac{n}{2} ; \frac{p^{2}}{m^{2}}\right)
$$

where $F(a, b ; c ; z)$ is the hypergeometric function [53]. In order to discuss the power counting properties of $H$ (in the chiral limit), we make use of Eq. 15.3.6 of Ref. [53] to rewrite Eq. (13) as

$$
\begin{aligned}
H\left(p^{2}, m^{2}, 0 ; n\right)= & \frac{m^{n-4}}{(4 \pi)^{\frac{n}{2}}}\left[\frac{\Gamma\left(2-\frac{n}{2}\right)}{n-3} F\left(1,2-\frac{n}{2} ; 4-n ;-\Delta\right)\right. \\
& \left.+(-\Delta)^{n-3} \Gamma\left(\frac{n}{2}-1\right) \Gamma(3-n) F\left(\frac{n}{2}-1, n-2 ; n-2 ;-\Delta\right)\right] .
\end{aligned}
$$

Making use of

$$
F(a, b ; c ; z)=1+\frac{a b}{c} z+\frac{a(a+1) b(b+1)}{c(c+1)} \frac{z^{2}}{2}+\cdots
$$

for $|z|<1$ and the fact that $\Delta$ counts as a small quantity of order $\mathcal{O}(q)$, we immediately see that the first term of Eq. (14) contains a contribution which does not satisfy the above power counting, i.e., which is not proportional to $\mathcal{O}(q)$ as $n \rightarrow 4$. Using the expansion of Eq. (15) together with $\Gamma(1+x)=x \Gamma(x)$ we obtain, as $n \rightarrow 4$,

$$
H=\frac{m^{n-4}}{(4 \pi)^{\frac{n}{2}}}\left[\frac{\Gamma\left(2-\frac{n}{2}\right)}{n-3}+\left(1-\frac{p^{2}}{m^{2}}\right) \ln \left(1-\frac{p^{2}}{m^{2}}\right)+\left(1-\frac{p^{2}}{m^{2}}\right)^{2} \ln \left(1-\frac{p^{2}}{m^{2}}\right)+\cdots\right]
$$

where $\cdots$ refers to terms which are at least of order $\mathcal{O}\left(q^{3}\right)$ or $O(n-4) \cdot{ }^{3}$ If we subtract

$$
\frac{m^{n-4}}{(4 \pi)^{\frac{n}{2}}} \frac{\Gamma\left(2-\frac{n}{2}\right)}{n-3}
$$

from Eq. (16) we obtain as the renormalized integral

$$
H_{R}\left(p^{2}, m^{2}, 0 ; n\right)=\frac{m^{n-4}}{(4 \pi)^{n / 2}}\left[\left(1-\frac{p^{2}}{m^{2}}\right) \ln \left(1-\frac{p^{2}}{m^{2}}\right)+\left(1-\frac{p^{2}}{m^{2}}\right)^{2} \ln \left(1-\frac{p^{2}}{m^{2}}\right)+\cdots\right] .
$$

The subtracted term of Eq. (17) is local in the external momentum $p$, i.e., it is a polynomial in $p^{2}$ and can thus be obtained by a finite number of counterterms in the most general effective Lagrangian. In other words, using an ordinary subtractive renormalization with an appropriately chosen renormalization condition we obtained the renormalized expression of Eq. (18) which satisfies the power counting discussed above.

Using the example of Eq. (6) (in the chiral limit) we now apply a conventional renormalization prescription which allows us to identify those terms which we subtract from a given integral without explicitly calculating the integral beforehand. In essence we work with a

${ }^{3}$ Note that we count a term of the type $-\Delta \ln (-\Delta)$ as $\mathcal{O}(q)$. 
modified integrand which is obtained from the original integrand by subtracting a suitable number of counterterms. ${ }^{4}$ The meaning of suitable in the present context will be explained in a moment. To that end we consider the series

$$
\begin{aligned}
& \sum_{l=0}^{\infty} \frac{\left(p^{2}-m^{2}\right)^{l}}{l !}\left[\left(\frac{1}{2 p^{2}} p_{\mu} \frac{\partial}{\partial p_{\mu}}\right)^{l} \frac{1}{\left(k^{2}+i 0^{+}\right)\left[k^{2}-2 k \cdot p+\left(p^{2}-m^{2}\right)+i 0^{+}\right]}\right]_{p^{2}=m^{2}} \\
= & \left.\frac{1}{\left(k^{2}+i 0^{+}\right)\left(k^{2}-2 k \cdot p+i 0^{+}\right)}\right|_{p^{2}=m^{2}} \\
& +\left(p^{2}-m^{2}\right)\left[\frac{1}{2 m^{2}} \frac{1}{\left(k^{2}-2 k \cdot p+i 0^{+}\right)^{2}}-\frac{1}{2 m^{2}} \frac{1}{\left(k^{2}+i 0^{+}\right)\left(k^{2}-2 k \cdot p+i 0^{+}\right)}\right. \\
& \left.-\frac{1}{\left(k^{2}+i 0^{+}\right)\left(k^{2}-2 k \cdot p+i 0^{+}\right)^{2}}\right]_{p^{2}=m^{2}} \\
& +\cdots,
\end{aligned}
$$

where $[\cdots]_{p^{2}=m^{2}}$ means that we consider the coefficients of $\left(p^{2}-m^{2}\right)^{l}$ only for four-momenta $p^{\mu}$ satisfying the on-mass-shell condition. ${ }^{5}$ Although the coefficients still depend on the direction of $p^{\mu}$, after integration of this series with respect to the loop momentum $k$ and evaluation of the resulting coefficients for $p^{2}=m^{2}$, the integrated series is a function of $p^{2}$ only. In fact, as was shown in Ref. [54], the integrated series exactly reproduces the first term of Eq. (14). At this point we stress that

$$
-\left.i \int \frac{d^{n} k}{(2 \pi)^{n}} \frac{1}{\left(k^{2}+i 0^{+}\right)\left(k^{2}-2 k \cdot p+i 0^{+}\right)}\right|_{p^{2}=m^{2}}
$$

and

$$
\left[-i \int \frac{d^{n} k}{(2 \pi)^{n}} \frac{1}{\left(k^{2}+i 0^{+}\right)\left(k^{2}-2 k \cdot p+p^{2}-m^{2}+i 0^{+}\right)}\right]_{p^{2}=m^{2}}
$$

are not the same for $n \leq 3$. Let us provide a formal definition of our renormalization scheme: we subtract from the integrand of $H\left(p^{2}, m^{2}, 0 ; n\right)$ those terms of the series of Eq. (19) which violate the power counting. These terms are always analytic in the small parameter and do not contain infrared singularities. In the above example we only need to subtract the first term. All the higher-order terms contain infrared singularities. For example, the last term of the second coefficient would generate a behavior $k^{3} / k^{4}$ of the integrand for $n=4$. The integral of the first term of Eq. (19) is given by Eq. (17), and we end up with Eq. (18) for the renormalized integral. Since we make use of the subtraction point $p^{2}=m^{2}$, we denote our renormalization condition "extended on-mass-shell" (EOMS) scheme in analogy with the on-mass-shell renormalization scheme in renormalizable theories.

Let us now compare with the approach of Becher and Leutwyler of Ref. 23], where the integral $H$ is divided into the so-called infrared (singular) part $I$ and the remainder $R$, defined as

$$
I \equiv \kappa(m ; n) \int_{0}^{\infty} d z[C(z, \Delta)]^{\frac{n}{2}-2}
$$

\footnotetext{
${ }^{4}$ In the often used zero-momentum subtraction scheme a Taylor series expansion of the integrand with respect to the external momentum $p^{\mu}$ around $p^{\mu}=0$ is used.

5 Equation (19) is not a Taylor series of the integrand.
} 


$$
R \equiv-\kappa(m ; n) \int_{1}^{\infty} d z[C(z, \Delta)]^{\frac{n}{2}-2}
$$

The analytical expressions for both integrals are given by ${ }^{6}$

$$
\begin{aligned}
I & =\frac{m^{n-4}}{(4 \pi)^{\frac{n}{2}}}(-\Delta)^{n-3} \Gamma\left(\frac{n}{2}-1\right) \Gamma(3-n) \frac{1}{(1+\Delta)^{\frac{n}{2}-1}}, \\
R & =-\kappa(m ; n)(1+\Delta)^{\frac{n}{2}-2} \frac{\Gamma(3-n)}{\Gamma(4-n)} F\left(2-\frac{n}{2}, 3-n ; 4-n ; \frac{\Delta}{1+\Delta}\right) .
\end{aligned}
$$

Let us discuss a few properties of $I$ and $R$, respectively. Counting $\Delta$ as a small quantity of $\mathcal{O}(q)$, the infrared part $I$ respects a simple power counting by being proportional to $q^{n-3}$. As $n \rightarrow 4, I$ cannot be expanded in a power series in $\Delta$, because

$$
(-\Delta)^{n-3} \Gamma(3-n)=-\Delta\left[\frac{1}{n-4}-\Gamma^{\prime}(1)-1\right]-\Delta \ln (-\Delta)+O(n-4) .
$$

Finally, for noninteger values of $n, I$ contains noninteger powers of $\Delta$. On the other hand, due to the analytic properties of the hypergeometric function, the remainder $R$ can be expanded in an ordinary Taylor series in $\Delta$ even for noninteger values of $n$. However, as $\Delta \rightarrow 0, R$ does not fit into the above power counting, i.e., it is not proportional to a small quantity of order $q$ raised to the power $n-3$. In the approach of Becher and Leutwyler one explicitly keeps the contribution $I$ of $H$ (with subtracted singularities when $n$ approaches 4 ) as the result of the integral and drops $R$ arguing that it is effectively taken into account through an infinite number of counterterms in the most general effective Lagrangian. As pointed out in Ref. [23], the infrared part $I$ also contains an infinite number of divergent terms if expanded in powers of $\Delta$. An infinite number of divergent terms in $R$ and $I$ exactly cancel each other and one is left with one ultraviolet divergent term in $H$ which is $\Delta$ independent, namely Eq. (17).

\section{B. Finite pion mass}

We now generalize our renormalization scheme to the case of a nonvanishing pion mass [see Eq. (6) ]. For easier comparison with Ref. [23] we introduce the variables

$$
\Omega=\frac{p^{2}-m^{2}-M^{2}}{2 m M}, \quad \alpha=\frac{M}{m},
$$

where $\Omega$ counts as $\mathcal{O}\left(q^{0}\right)$ for $p^{2} \neq m^{2}\left[\mathcal{O}(q)\right.$ for $\left.p^{2}=m^{2}\right]$ and $\alpha$ counts as $\mathcal{O}(q)$. We obtain for Eq. (10), as $n \rightarrow 4$,

$$
H=-2 \bar{\lambda}+\frac{1}{16 \pi^{2}}-\frac{1}{8 \pi^{2}} \frac{\alpha \sqrt{1-\Omega^{2}}}{1+2 \alpha \Omega+\alpha^{2}} \arccos (-\Omega)-\frac{1}{8 \pi^{2}} \frac{\alpha(\alpha+\Omega)}{1+2 \alpha \Omega+\alpha^{2}} \ln (\alpha),
$$

where

$$
\bar{\lambda}=\frac{m^{n-4}}{(4 \pi)^{2}}\left\{\frac{1}{n-4}-\frac{1}{2}\left[\ln (4 \pi)+\Gamma^{\prime}(1)+1\right]\right\}
$$

\footnotetext{
${ }^{6}$ The correct imaginary parts are obtained by replacing $m^{2} \rightarrow m^{2}-i 0^{+}$.
} 
Clearly, the first two terms of Eq. (25) violate our power counting, since we want the renormalized integral to be of $\mathcal{O}(q)$ as $n \rightarrow 4$.

In order to apply our renormalization scheme to Eq. (6), we observe that the dimensionally regularized integral contains a part which, for noninteger $n$, is proportional to noninteger powers of $M$ but does not violate the power counting. On the other hand, the remaining piece of the integral may always, i.e., for arbitrary $n$, be expanded in non-negative powers of $M$, and it is this contribution which is responsible for the violation of power counting. We expand this second part in terms of $M$ and $p^{2}-m^{2}$ and subtract those terms which violate the power counting. In practice, we realize this scheme by writing down a series similar to Eq. (19), where, in addition, we expand pion propagators in powers of $M^{2}$. In the present case we only need to subtract the first term to satisfy the power counting:

$$
H_{\text {subtr }}=-\left.i \int \frac{d^{n} k}{(2 \pi)^{n}} \frac{1}{k^{2}+i 0^{+}} \frac{1}{k^{2}-2 p \cdot k+i 0^{+}}\right|_{p^{2}=m^{2}}=-2 \bar{\lambda}+\frac{1}{16 \pi^{2}}+O(n-4) .
$$

Subtracting Eq. (27) from Eq. (25) our final expression for the renormalized integral reads

$$
H_{R}=-\frac{1}{8 \pi^{2}} \frac{\alpha \sqrt{1-\Omega^{2}}}{1+2 \alpha \Omega+\alpha^{2}} \arccos (-\Omega)-\frac{1}{8 \pi^{2}} \frac{\alpha(\alpha+\Omega)}{1+2 \alpha \Omega+\alpha^{2}} \ln (\alpha) .
$$

Again, the subtraction term $H_{\text {subtr }}$ of Eq. (27) is local in the external momenta and can thus be realized as a counterterm in the most general effective Lagrangian. Let us stress one more time that we count a term $\alpha \ln (\alpha)$ as $\mathcal{O}(q)$. Moreover, when expanded in small quantities, $H_{R}$ consists of an infinite string of terms of $\mathcal{O}\left(q^{l}\right)$ with $l \geq 1$. In other words, when we say that an expression is of $\mathcal{O}(q)$, we refer to the minimal power $q^{1}$ of that expression. This situation has to be contrasted with the mesonic sector, where an expression of, say, $\mathcal{O}\left(q^{4}\right)$ exclusively consists of terms of $\mathcal{O}\left(q^{4}\right)$. We conclude that using ordinary renormalization with appropriately chosen renormalization conditions allows us to obtain the renormalized expression of Eq. (28) which satisfies power counting.

It is instructive to compare Eq. (28) with the result of the infrared regularization of Becher and Leutwyler, where the integral $H$ is divided into an infrared part $I$ and a remainder $R$ [23]:

$$
\begin{aligned}
I= & \bar{\lambda} \nu-\frac{1}{8 \pi^{2}} \frac{\alpha \sqrt{1-\Omega^{2}}}{1+2 \alpha \Omega+\alpha^{2}} \arccos \left(-\frac{\alpha+\Omega}{\sqrt{1+2 \alpha \Omega+\alpha^{2}}}\right) \\
& -\frac{1}{16 \pi^{2}} \frac{\alpha(\alpha+\Omega)}{1+2 \alpha \Omega+\alpha^{2}}[2 \ln (\alpha)-1] \\
R= & -(2+\nu) \bar{\lambda}+\frac{1}{8 \pi^{2}} \frac{\alpha \sqrt{1-\Omega^{2}}}{1+2 \alpha \Omega+\alpha^{2}} \arcsin \left(\frac{\alpha \sqrt{1-\Omega^{2}}}{\sqrt{1+2 \alpha \Omega+\alpha^{2}}}\right)+\frac{1}{16 \pi^{2}} \frac{1+\alpha \Omega}{1+2 \alpha \Omega+\alpha^{2}}(30
\end{aligned}
$$

where

$$
\nu=-\frac{p^{2}-m^{2}+M^{2}}{p^{2}}
$$

Using elementary relations among the inverse trigonometric functions, the sum of $I$ and $R$ is indeed identical to Eq. (25). In this decomposition $I$ satisfies the power counting whereas $R$, violating the power counting, is absorbed into an infinite number of counterterms. The first (infinite) term of $I$ is also taken care of by renormalization. 


\section{NUCLEON SELF-ENERGY}

As a specific example, we will now turn to the calculation of the nucleon self-energy at $\mathcal{O}\left(q^{4}\right)$. The complete propagator of the nucleon is defined as the Fourier transform

$$
S_{0}(p)=\int d^{4} x e^{i p \cdot x} S_{0}(x)
$$

of the two-point function

$$
S_{0}(x)=-i\left\langle 0\left|T\left[\Psi_{0}(x) \bar{\Psi}_{0}(0)\right]\right| 0\right\rangle,
$$

where $\Psi_{0}$ denotes the bare nucleon field. We parametrize

$$
S_{0}(p)=\frac{1}{\not p-m_{0}-\Sigma_{0}(\not p)} \equiv \frac{1}{\not p-m-\Sigma(\not p)}
$$

where $m_{0}$ refers to the bare mass of Eq. (A1), whereas $m$ is the nucleon pole mass in the chiral limit. Here, $\Sigma_{0}(\not p)$ and $\Sigma(\not p)$ are matrix functions [55] which, using $\not p \not p=p^{2}$, can be parametrized as

$$
\Sigma_{0}(x)=-x f_{0}\left(x^{2}\right)+m_{0} g_{0}\left(x^{2}\right)
$$

with an analogous expression for $\Sigma$.

We will express the nucleon self-energy $\Sigma(\not p)$ in terms of $m$, the lowest-order pion mass $M$, and bare coupling constants. In terms of Feynman diagrams, $-i \Sigma(\not p)$ represents the oneparticle-irreducible perturbative contribution to the two-point function. Moreover, it also contains contributions of counterterms generated by $m_{0}$, which make sure that the pole mass in the chiral limit, $m$, stays put. However, for the sake of simplicity we will not explicitly show these counterterms.

As usual the physical nucleon mass is defined through the pole of the full propagator at $\not p=m_{N}$,

$$
m_{N}-m_{0}-\Sigma_{0}\left(m_{N}\right)=m_{N}-m-\Sigma\left(m_{N}\right)=0
$$

while the wave function renormalization constant $Z_{0}$ is defined as the residue at $\not p=m_{N}$,

$$
\begin{aligned}
S_{0}(p) & =\frac{1}{\not p-m_{0}-\Sigma_{0}\left(m_{N}+\not p-m_{N}\right)} \\
& =\frac{1}{\not p-m_{0}-\Sigma_{0}\left(m_{N}\right)-\left(\not p-m_{N}\right) \Sigma_{0}^{\prime}\left(m_{N}\right)+O\left[\left(\not p-m_{N}\right)^{2}\right]} \\
& =\frac{1}{\left(\not p-m_{N}\right)\left[1-\Sigma_{0}^{\prime}\left(m_{N}\right)+O\left(\not p-m_{N}\right)\right]} \\
& \rightarrow \frac{Z_{0}}{\not p-m_{N}+i 0^{+}} \text {for } \not p \rightarrow m_{N},
\end{aligned}
$$

yielding

$$
Z_{0}=\frac{1}{1-\Sigma_{0}^{\prime}\left(m_{N}\right)}=\frac{1}{1-\Sigma^{\prime}\left(m_{N}\right)} .
$$

At $\mathcal{O}\left(q^{4}\right)$, the self-energy receives contact contributions from $\mathcal{L}_{\pi N}^{(2)}$ and $\mathcal{L}_{\pi N}^{(4)}$ as well as the one-loop contributions of Fig. 1.

$$
\Sigma=\Sigma_{\text {contact }}+\Sigma_{\text {loop }} .
$$


The contact contributions read

$$
\Sigma_{\text {contact }}=-4 M^{2} c_{1}^{0}-2 M^{4}\left(8 e_{38}^{0}+e_{115}^{0}+e_{116}^{0}\right),
$$

where the superscripts 0 refer to bare parameters. Applying Feynman rules we obtain three one-loop contributions (see Fig. (1)

$$
\Sigma_{\text {loop }}=\Sigma_{a}+\Sigma_{b}+\Sigma_{c}
$$

where

$$
\begin{aligned}
\Sigma_{a} & =\frac{3{\stackrel{\circ}{A_{0}}}^{2}}{4 F_{0}^{2}} i \int \frac{d^{n} k}{(2 \pi)^{n}} \not k \gamma_{5} \frac{1}{\not p-\not k-m+i 0^{+}} \not k \gamma_{5} \frac{1}{k^{2}-M^{2}+i 0^{+}} \\
& =\frac{3 \stackrel{\circ}{A 0}^{2}}{4 F_{0}^{2}} i \int \frac{d^{n} k}{(2 \pi)^{n}} \frac{\not k(\not p-\not k-m) \not k}{(k-p)^{2}-m^{2}+i 0^{+}} \frac{1}{k^{2}-M^{2}+i 0^{+}}, \\
\Sigma_{b} & =-4 M^{2} c_{1}^{0} \frac{3 g_{A_{0}}^{\circ}}{4 F_{0}^{2}} i \int \frac{d^{n} k}{(2 \pi)^{n}} \not k \gamma_{5}\left(\frac{1}{\not p-\not k-m+i 0^{+}}\right)^{2} \not k \gamma_{5} \frac{1}{k^{2}-M^{2}+i 0^{+}} \\
& =-4 M^{2} c_{1}^{0} \frac{\partial \Sigma_{a}}{\partial m}, \\
\Sigma_{c} & =3 \frac{M^{2}}{F_{0}^{2}}\left(2 c_{1}^{0}-c_{3}^{0}-\frac{p^{2}}{m^{2}} \frac{c_{2}^{0}}{n}\right) i \int \frac{d^{n} k}{(2 \pi)^{n}} \frac{1}{k^{2}-M^{2}+i 0^{+}} .
\end{aligned}
$$

Using $\left\{\gamma^{\mu}, \gamma^{\nu}\right\}=2 g^{\mu \nu}$, Eq. (39) can be expressed in terms of the basis integrals of Appendix B as

$$
\begin{aligned}
\Sigma_{a}= & -\frac{3{\stackrel{\circ}{A_{0}}}^{2}}{4 F_{0}^{2}}\left\{(\not p+m) I_{N}+M^{2}(\not p+m) I_{N \pi}(-p, 0)\right. \\
& \left.-\frac{\left(p^{2}-m^{2}\right) \not p p}{2 p^{2}}\left[\left(p^{2}-m^{2}+M^{2}\right) I_{N \pi}(-p, 0)+I_{N}-I_{\pi}\right]\right\} .
\end{aligned}
$$

The renormalization of the loop diagrams is performed in two steps. First we render the diagrams finite by applying the subtraction scheme used by Gasser and Leutwyler [13, 16] which we denote by the modified minimal subtraction scheme of ChPT $(\widetilde{\mathrm{MS}}){ }^{7}$ We choose the renormalization parameter (unit of mass or 't Hooft parameter) $\mu=m$. In a second step we then perform additional finite subtractions for integrals which contain nucleon propagators with the purpose of imposing our power counting scheme. In fact, in order to apply the MS subtraction in practical calculations, we do not actually need to explicitly write down the corresponding counterterms. We simply subtract all loop diagrams and replace the bare couplings with the couplings corresponding to the $\widetilde{\mathrm{MS}}$ scheme. In the above expressions we replace subscripts and superscripts "0" denoting bare coupling constants with "r" and

\footnotetext{
${ }^{7}$ In distinction to the $\overline{\mathrm{MS}}$ scheme commonly used in Standard Model calculations, the $\widetilde{\mathrm{MS}}$ scheme contains an additional finite subtraction term. To be specific, in $\widetilde{\mathrm{MS}}$ one uses multiples of $1 /(n-4)-$ $\left[\ln (4 \pi)+\Gamma^{\prime}(1)+1\right] / 2$ instead of $1 /(n-4)-\left[\ln (4 \pi)+\Gamma^{\prime}(1)\right] / 2$ in $\overline{\mathrm{MS}}$.
} 
supply the integrals with indicators "r" referring to the fact that they have been subtracted. For example, the result for $\Sigma_{r, a}$ then reads

$$
\begin{aligned}
\Sigma_{r, a}= & -\frac{3{\stackrel{\circ}{A_{r}}}^{2}}{4 F_{r}^{2}}\left\{M^{2}(\not p+m) I_{N \pi}^{r}(-p, 0)\right. \\
& \left.-\frac{\left(p^{2}-m^{2}\right) \not p}{2 p^{2}}\left[\left(p^{2}-m^{2}+M^{2}\right) I_{N \pi}^{r}(-p, 0)-I_{\pi}^{r}\right]\right\},
\end{aligned}
$$

where the expressions for $I_{\pi}^{r}$ and $I_{N \pi}^{r}$ are given in Eqs. (B4) and (B8) of Appendix B.

The $\widetilde{\mathrm{MS}}$-subtracted self-energy corresponds to the Green function

$$
S_{1}(p)=\int d^{4} x e^{i p \cdot x} S_{1}(x)
$$

where

$$
S_{1}(x)=-i\left\langle 0\left|T\left[\Psi_{1}(x) \bar{\Psi}_{1}(0)\right]\right| 0\right\rangle
$$

is the two-point function of the $\widetilde{\mathrm{MS}}$-renormalized field

$$
\Psi_{1}(x) \equiv \Psi_{\widetilde{\mathrm{MS}}}(x)=\frac{\Psi_{0}(x)}{\sqrt{Z_{01}}}
$$

We refer to $\sqrt{Z_{01}}$ as the field redefinition constant [see Eq. (A2)] connecting the bare field $\Psi_{0}$ and the $\widetilde{\mathrm{MS}}$-renormalized field $\Psi_{1}$. Analogous to Eq. (34), the physical nucleon mass is determined through the pole of the $\widetilde{\mathrm{MS}}$-renormalized propagator. We obtain for the mass in the $\widetilde{\mathrm{MS}}$ scheme

$$
\begin{aligned}
m_{N}= & m-4 c_{1}^{r} M^{2}+\frac{3{\stackrel{\circ}{A_{r}}}^{2} M^{2}}{32 \pi^{2} F_{r}^{2}} m\left(1+8 c_{1}^{r} m\right)-\frac{3{\stackrel{\circ}{A_{r}}}^{2} M^{3}}{32 \pi F_{r}^{2}} \\
& +\frac{3 M^{4}}{32 \pi^{2} F_{r}^{2}} \ln \left(\frac{M}{m}\right)\left(8 c_{1}^{r}-c_{2}^{r}-4 c_{3}^{r}-\frac{{\stackrel{\circ}{A_{r}}}^{2}}{m}\right) \\
& +\frac{3{\stackrel{\circ}{A_{r}}}^{2} M^{4}}{32 \pi^{2} F_{r}^{2} m}\left[1+4 c_{1}^{r} m\right] \\
& +M^{4}\left(\frac{3}{128 \pi^{2} F_{r}^{2}} c_{2}^{r}-16 e_{38}^{r}-2 e_{115}^{r}-2 e_{116}^{r}\right)+O\left(M^{5}\right),
\end{aligned}
$$

where "r" refers to $\widetilde{\mathrm{MS}}$-renormalized quantities. When solving Eq. (34) in terms of Eqs. (37) and (38), we consistently omitted terms which count as $O\left(\hbar^{2}\right)$ in the loop expansion, i.e., terms proportional to $\left(\stackrel{\circ}{g}_{A_{0}} / F_{0}\right)^{4}$, as well as terms proportional to $\left(c_{1}^{r}\right)^{2}$ which do not contribute in our final extended on-mass-shell expression for the nucleon mass.

Correspondingly, the wave function renormalization constant of the $\widetilde{\mathrm{MS}}-\mathrm{renormalized}$ propagator,

$$
S_{1}(p)=\frac{1}{\not p-m-\Sigma_{r}(\not p)} \rightarrow \frac{Z_{1}}{\not p-m_{N}+i 0^{+}} \text {for } \not p \rightarrow m_{N},
$$


is an expression of $\mathcal{O}\left(q^{3}\right),{ }^{8}$ given by

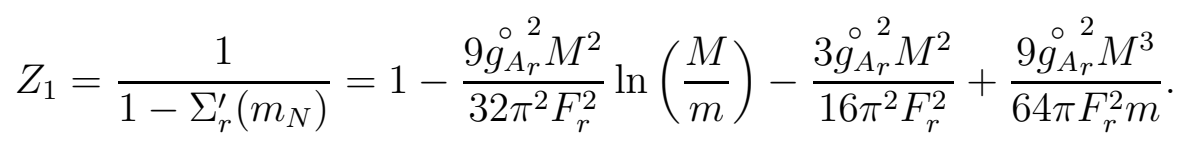

Clearly, we do not require that the propagators of renormalized fields have unit residue at the physical pole mass [12, 55]. The relation between $Z_{1}$ on the one hand and $Z_{0}$ and the field redefinition constant $\sqrt{Z_{01}}$ on the other hand is given by

$$
Z_{1}=\frac{Z_{0}}{Z_{01}}
$$

Note, in particular, that $Z_{1}$ is finite, whereas both $Z_{0}$ and $Z_{01}$ contain infinities resulting from ultraviolet divergences.

In order to perform the second step, namely another finite renormalization, a given $\widetilde{\mathrm{MS}}$ renormalized diagram is written as the sum of a subtracted diagram which, through the application of the subtraction scheme described in the previous sections, satisfies the power counting and a remainder which violates the power counting and thus still needs to be subtracted. We expand the finite renormalized couplings of the $\widetilde{\mathrm{MS}}$ scheme in a series in terms of couplings of our generalized on-mass-shell scheme. In doing so, we generate finite counterterms, responsible for additional finite subtractions. These counterterms are fixed so that the net result of combining the counterterm diagrams with those parts of the MS-renormalized diagrams which violate the power counting are of the same order as the subtracted diagram. (Note that depending on the applied renormalization condition the net result may vanish.) Hence the sum of an $\widetilde{\mathrm{MS}}$-renormalized diagram and the corresponding counterterm diagram satisfies the power counting.

For the case at hand, we determine the terms to be subtracted from $\Sigma_{a}$ by first expanding the integrands and coefficients in Eq. (42) in powers of $M^{2}, \not p-m$ and $p^{2}-m^{2}$. In this expansion we keep all the terms having a chiral order which is smaller than what is suggested by the power counting for the given diagram. We then obtain

$$
\Sigma_{r, a+b}^{\mathrm{subtr}}=\frac{3{\stackrel{\circ}{A_{r}}}^{2}}{32 \pi^{2} F_{r}^{2}}\left[m M^{2}-\frac{\left(p^{2}-m^{2}\right)^{2}}{4 m}\right]+\frac{3 c_{1}^{r} \stackrel{\circ}{g_{A r}^{2}} M^{2}}{8 \pi^{2} F_{r}^{2}}\left[m(\not p+m)-\frac{3}{2}\left(p^{2}-m^{2}\right)\right] .
$$

Equation (48) specifies the part of the self-energy diagram which has to be subtracted. We fix the corresponding counterterms so that they exactly cancel the expression given by Eq. (48). Since the most general Lagrangian contains all the structures consistent with the symmetries of the theory, it also provides the required counterterms. Finally, the renormalized selfenergy expression is obtained by subtracting Eq. (48) from the MS-subtracted version of Eqs. (39) and (40) and replacing the $\widetilde{\mathrm{MS}}$-renormalized couplings with the ones of our generalized on-mass-shell scheme. We note that the $\widetilde{\mathrm{MS}}$-subtracted version for $\Sigma_{c}$ needs no further subtraction because it is already of order $\mathcal{O}\left(q^{4}\right)$.

The correction to the nucleon mass resulting from the counterterms is calculated by substituting $\not p=m_{N}$ into the negative of Eq. (48). [Recall that Eq. (48) has to be subtracted.]

\footnotetext{
8 The reduction by one chiral order in comparison with the self-energy can be understood in terms of the derivative in the definition of the wave function renormalization constant.
} 
We thus obtain the following expression for the contribution to the mass:

$$
\Delta m=-\frac{3 \stackrel{\circ}{A}^{2} M^{2}}{32 \pi^{2} F^{2}}\left(m+8 c_{1} m^{2}\right) .
$$

Finally, we express the physical mass of the nucleon up to and including order $q^{4}$ as $[56,57]^{9}$

$$
m_{N}=m+k_{1} M^{2}+k_{2} M^{3}+k_{3} M^{4} \ln \left(\frac{M}{m}\right)+k_{4} M^{4}+O\left(M^{5}\right)
$$

where $m$ is the nucleon mass in the chiral limit and $M^{2}=2 B \hat{m}$ is the leading-order result for $M_{\pi}^{2}$. In terms of the EOMS-renormalized parameters, the coefficients $k_{i}$ are then given by

$$
\begin{aligned}
& k_{1}=-4 c_{1} \\
& k_{2}=-\frac{3 \stackrel{\circ}{A}^{2}}{32 \pi F^{2}}, \\
& k_{3}=\frac{3}{32 \pi^{2} F^{2}}\left(8 c_{1}-c_{2}-4 c_{3}-\frac{\stackrel{\circ}{A}^{2}}{m}\right), \\
& k_{4}=\frac{3 \stackrel{\circ}{A}^{2}}{32 \pi^{2} F^{2} m}\left(1+4 c_{1} m\right)+\frac{3}{128 \pi^{2} F^{2}} c_{2}-16 e_{38}-2 e_{115}-2 e_{116} .
\end{aligned}
$$

Comparing with Ref. 23], we see that the lowest-order correction ( $k_{1}$ term) and those terms which are nonanalytic in the quark mass $\hat{m}$ ( $k_{2}$ and $k_{3}$ terms) coincide. On the other hand, the analytic $k_{4}$ term $\left(\sim M^{4}\right)$ is different. This is not surprising, because we use a different renormalization scheme and hence the difference between the two results is compensated by different values of the renormalized parameters.

The contribution of the counterterms in Eq. (49) to the physical mass is generated by the following expansion of the coupling $c_{1}^{r}$ in terms of our renormalized parameters:

$$
c_{1}^{r}=c_{1}+\frac{3 m \stackrel{\circ}{A}^{2}}{128 \pi^{2} F^{2}}\left[1+8 m c_{1}\right]+\cdots,
$$

while the net result of the contributions of the counterterms which are generated by expanding the other parameters vanishes at the given order. Finally, the wave function renormalization (residue at the pole) does not obtain a contribution from counterterms at this order so that $Z$ for our renormalization scheme reads

$$
Z=1-\frac{9{\stackrel{\circ}{g_{A}}}^{2} M^{2}}{32 \pi^{2} F^{2}} \ln \left(\frac{M}{m}\right)-\frac{3 \stackrel{\circ}{A}^{2} M^{2}}{16 \pi^{2} F^{2}}+\frac{9_{g_{A}}^{2} M^{3}}{64 \pi F^{2} m}
$$

\section{CONCLUSIONS}

We have discussed a new renormalization scheme which allows for a simple and consistent power counting in the single-nucleon sector of relativistic chiral perturbation theory. In

\footnotetext{
${ }^{9}$ In our convention $k_{3}$ is larger by a factor of two than in Refs. [56, 57], because we use $\ln (M / m)$ instead of $\ln \left(M^{2} / m^{2}\right)$.
} 
order to renormalize a given diagram, using Eq. (7) one first assigns a chiral order $D$ to that diagram. Applying standard techniques the diagram is reduced to the sum of dimensionally regularized scalar integrals multiplied by corresponding Dirac structures. By expanding the integrands as well as the coefficients in small quantities one identifies those terms which need to be subtracted in order to produce the renormalized diagram with the chiral order $D$ determined beforehand. It is this aspect which we refer to as "appropriately chosen renormalization conditions," because these subtractions can be realized in terms of local counterterms in the most general effective Lagrangian. For pedagogical reasons we have performed the subtractions in two steps: the first step, namely, applying a modified minimal subtraction scheme (of ChPT) to get rid of the ultraviolet divergences, corresponds to the procedure used by Gasser, Sainio, and Švarc [16]. In a second step we have then performed additional finite subtractions for those integrals which contain nucleon propagators such that the subtracted diagram satisfies our power counting scheme. We have explicitly applied our scheme to a calculation of the nucleon mass. Comparing with the results of the infrared regularization method [23] we have seen that the expressions for the nucleon mass in the two schemes only differ by terms which are analytic in the quark masses. These findings are consistent, because such terms are renormalization-scheme dependent.

Finally, our renormalization scheme is neither restricted to the single-nucleon sector nor to the interaction of Goldstone bosons with fermions. For example, it may also be used in the NN sector or for describing the interaction of vector and axial-vector mesons [58]. In conclusion, we have presented a simple renormalization scheme which produces a consistent power counting for relativistic baryon chiral perturbation theory.

\section{Acknowledgments}

The work of T.F. and S.S. was supported by the Deutsche Forschungsgemeinschaft (SFB 443). J.G. acknowledges the support of the Alexander von Humboldt Foundation. G.J. was supported by NSF grant OPP-0236449/G067771.

\section{APPENDIX A: THE GENERATION OF COUNTERTERMS}

The renormalization of the effective field theory (of pions and nucleons) is performed by expressing all the bare parameters and bare fields of the effective Lagrangian in terms of renormalized quantities [12]. In this process, one generates counterterms which are responsible for the absorption of all the divergences occurring in the calculation of loop diagrams. In

order to illustrate the procedure let us discuss $\mathcal{L}_{\pi N}^{(1)}$ and consider the free part in combination with the $\pi N$ interaction term with the smallest number of pion fields,

$$
\mathcal{L}_{\pi N}^{(1)}=\bar{\Psi}_{0}\left(i \gamma_{\mu} \partial^{\mu}-m_{0}-\frac{1}{2} \frac{\stackrel{\circ}{g_{A_{0}}}}{F_{0}} \gamma_{\mu} \gamma_{5} \tau^{a} \partial^{\mu} \pi_{0}^{a}\right) \Psi_{0}+\cdots,
$$

given in terms of bare fields and parameters denoted by subscripts 0 . Introducing renormalized fields (we work in the isospin-symmetric limit) through

$$
\Psi=\frac{\Psi_{0}}{\sqrt{Z_{\Psi}}}, \quad \pi^{a}=\frac{\pi_{0}^{a}}{\sqrt{Z_{\pi}}}
$$


we express the field redefinition constants $\sqrt{Z_{\Psi}}$ and $\sqrt{Z_{\pi}}$ and the bare quantities in terms of renormalized parameters:

$$
\begin{aligned}
Z_{\Psi} & =1+\delta Z_{\Psi}\left(m, \stackrel{\circ}{g}_{A}, g_{i}, \nu\right), \\
Z_{\pi} & =1+\delta Z_{\pi}\left(m, \circ_{A}, g_{i}, \nu\right), \\
m_{0} & =m(\nu)+\delta m\left(m, \stackrel{\circ}{g}_{A}, g_{i}, \nu\right), \\
{\stackrel{\circ}{A_{0}}} & =\stackrel{\circ}{g_{A}}(\nu)+\delta g_{A}\left(m, \stackrel{\circ}{g}_{A}, g_{i}, \nu\right),
\end{aligned}
$$

where $g_{i}, i=1, \cdots \infty$, collectively denote all the renormalized parameters which correspond to bare parameters $g_{i_{0}}$ of the full effective Lagrangian. The parameter $\nu$ indicates the dependence on the choice of the renormalization prescription. ${ }^{10}$ Substituting Eqs. (A2) and (A3) into Eq. (A1), we obtain

$$
\mathcal{L}_{\pi N}^{(1)}=\mathcal{L}_{\text {basic }}+\mathcal{L}_{\mathrm{ct}}+\cdots
$$

with the so-called basic and counterterm Lagrangians, respectively, ${ }^{11}$

$$
\begin{aligned}
\mathcal{L}_{\text {basic }} & =\bar{\Psi}\left(i \gamma_{\mu} \partial^{\mu}-m-\frac{1}{2} \frac{\stackrel{\circ}{A}_{A}}{F} \gamma_{\mu} \gamma_{5} \tau^{a} \partial^{\mu} \pi^{a}\right) \Psi \\
\mathcal{L}_{\mathrm{ct}} & =\delta Z_{\Psi} \bar{\Psi} i \gamma_{\mu} \partial^{\mu} \Psi-\delta\{m\} \bar{\Psi} \Psi-\frac{1}{2} \delta\left\{\frac{\stackrel{\circ}{A}_{A}}{F}\right\} \bar{\Psi} \gamma_{\mu} \gamma_{5} \tau^{a} \partial^{\mu} \pi^{a} \Psi
\end{aligned}
$$

where we introduced the abbreviations

$$
\begin{aligned}
\delta\{m\} & \equiv \delta Z_{\Psi} m+Z_{\Psi} \delta m, \\
\delta\left\{\frac{g_{A}}{F}\right\} & \equiv \delta Z_{\Psi} \frac{g_{A}}{F} \sqrt{Z_{\pi}}+Z_{\Psi}\left(\frac{\stackrel{\circ}{A}}{F_{0}}-\frac{\stackrel{\circ}{A}^{\prime}}{F}\right) \sqrt{Z_{\pi}}+\frac{\stackrel{\circ}{A}_{A}}{F}\left(\sqrt{Z_{\pi}}-1\right) .
\end{aligned}
$$

In Eq. (A5), $m, \stackrel{\circ}{A}_{A}$, and $F$ denote the chiral limit of the physical nucleon mass, the axialvector coupling constant, and the pion-decay constant, respectively. Expanding the counterterm Lagrangian of Eq. (A6) in powers of the renormalized coupling constants generates an infinite series, the individual terms of which are responsible for the subtraction of loop diagrams.

\section{APPENDIX B: LOOP INTEGRALS}

In this appendix we collect the loop integrals needed in the calculation of the nucleon mass. Most of them can be found in Ref. [23] or have been calculated using the method of

${ }^{10}$ Note that our choice $m(\nu)=m$, where $m$ is the nucleon pole mass in the chiral limit, is only one among an infinite number of possibilities.

${ }^{11}$ Reference [12] uses a slightly different convention which is obtained through the replacement $\left(\delta Z_{\Psi} m+\right.$ $\left.Z_{\Psi} \delta m\right) \rightarrow \delta m$. 
dimensional counting [54]. We use the following convention for scalar loop integrals:

$$
I_{N \cdots \pi \cdots}\left(p_{1}, \cdots, q_{1}, \cdots\right)=i \int \frac{d^{n} k}{(2 \pi)^{n}} \frac{1}{\left[\left(k+p_{1}\right)^{2}-m^{2}+i 0^{+}\right] \cdots\left[\left(k+q_{1}\right)^{2}-M^{2}+i 0^{+}\right] \cdots} \text {. }
$$

Tensor integrals are then derived in the standard fashion (see, for example, Appendix $\mathrm{C}$ of Ref. [15]). We do not display terms of $O(n-4)$ and higher. In what follows, $\bar{\lambda}$ is defined as

$$
\bar{\lambda}=\frac{m^{n-4}}{16 \pi^{2}}\left\{\frac{1}{n-4}-\frac{1}{2}\left[\ln (4 \pi)+\Gamma^{\prime}(1)+1\right]\right\} .
$$

From the set of purely pionic integrals we need

$$
I_{\pi}(q)=I_{\pi}(0)=I_{\pi}=i \int \frac{d^{n} k}{(2 \pi)^{n}} \frac{1}{k^{2}-M^{2}+i 0^{+}}=2 M^{2} \bar{\lambda}+\frac{M^{2}}{8 \pi^{2}} \ln \left(\frac{M}{m}\right) .
$$

The $\widetilde{M S}$-renormalized integral is obtained by simply dropping the term proportional to $\bar{\lambda}$ :

$$
I_{\pi}^{r}=\frac{M^{2}}{8 \pi^{2}} \ln \left(\frac{M}{m}\right)
$$

Next we consider the integral containing only a nucleon propapagator:

$$
I_{N}(p)=I_{N}(0)=I_{N}=i \int \frac{d^{n} k}{(2 \pi)^{n}} \frac{1}{k^{2}-m^{2}+i 0^{+}}=2 m^{2} \bar{\lambda} .
$$

The $\widetilde{\mathrm{MS}}$-renormalized integral then reads

$$
I_{N}^{r}=0
$$

Finally, we list the relevant integrals containing both a pion and a nucleon propagator:

$$
\begin{aligned}
I_{N \pi}(p, 0) & =i \int \frac{d^{n} k}{(2 \pi)^{n}} \frac{1}{\left[(k+p)^{2}-m^{2}+i 0^{+}\right]\left[k^{2}-M^{2}+i 0^{+}\right]} \\
& =2 \bar{\lambda}+\frac{1}{16 \pi^{2}}\left[-1+\frac{p^{2}-m^{2}+M^{2}}{p^{2}} \ln \left(\frac{M}{m}\right)+\frac{2 m M}{p^{2}} F(\Omega)\right],
\end{aligned}
$$

where

$$
F(\Omega)= \begin{cases}\sqrt{\Omega^{2}-1} \ln \left(-\Omega-\sqrt{\Omega^{2}-1}\right), & \Omega \leq-1 \\ \sqrt{1-\Omega^{2}} \arccos (-\Omega), & -1 \leq \Omega \leq 1 \\ \sqrt{\Omega^{2}-1} \ln \left(\Omega+\sqrt{\Omega^{2}-1}\right)-i \pi \sqrt{\Omega^{2}-1}, & 1 \leq \Omega\end{cases}
$$

with

$$
\Omega=\frac{p^{2}-m^{2}-M^{2}}{2 m M}
$$

Correspondingly,

$$
I_{N \pi}^{r}(p, 0)=I_{N \pi}(p, 0)-2 \bar{\lambda} .
$$


Furthermore we need

$$
\begin{aligned}
I_{N \pi}^{\mu}(-p, 0) & =i \int \frac{d^{n} k}{(2 \pi)^{n}} \frac{k^{\mu}}{\left[(k-p)^{2}-m^{2}+i 0^{+}\right]\left[k^{2}-M^{2}+i 0^{+}\right]} \\
& =\frac{p^{\mu}}{2 p^{2}}\left[\left(p^{2}-m^{2}+M^{2}\right) I_{N \pi}(-p, 0)+I_{N}-I_{\pi}\right] .
\end{aligned}
$$

[1] S. Weinberg, Physica A 96, 327 (1979).

[2] Y. Nambu, Phys. Rev. Lett. 4, 380 (1960).

[3] Y. Nambu and G. Jona-Lasinio, Phys. Rev. 122, 345 (1961); 124, 246 (1961).

[4] J. Goldstone, Nuovo Cimento 19, 154 (1961).

[5] J. Goldstone, A. Salam, and S. Weinberg, Phys. Rev. 127, 965 (1962).

[6] M. Gell-Mann, Physics (Long Island City, N.Y.) 1, 63 (1964).

[7] S. L. Adler and R. F. Dashen, Current Algebras and Applications to Particle Physics (Benjamin, New York, 1968).

[8] S. Treiman, R. Jackiw, and D. J. Gross, Lectures on Current Algebra and Its Applications (Princeton University Press, Princeton, 1972).

[9] V. de Alfaro, S. Fubini, G. Furlan, and C. Rossetti, Currents in Hadron Physics (NorthHolland, Amsterdam, 1973).

[10] S. Weinberg, Phys. Rev. Lett. 18, 188 (1967).

[11] S. Weinberg, The Quantum Theory Of Fields. Vol. 1: Foundations (Cambridge University Press, Cambridge, 1995).

[12] J. C. Collins, Renormalization (Cambridge University Press, Cambridge, UK, 1984).

[13] J. Gasser and H. Leutwyler, Ann. Phys. (N.Y.) 158, 142 (1984).

[14] J. Gasser and H. Leutwyler, Nucl. Phys. B250, 465 (1985).

[15] S. Scherer, in Advances in Nuclear Physics, Vol. 27, edited by J. W. Negele and E. W. Vogt (Kluwer Academic/Plenum Publishers, New York, 2003).

[16] J. Gasser, M. E. Sainio, and A. Švarc, Nucl. Phys. B307, 779 (1988).

[17] E. Jenkins and A. V. Manohar, Phys. Lett. B 255, 558 (1991); 259, 353 (1991).

[18] V. Bernard, N. Kaiser, J. Kambor, and U.-G. Meißner, Nucl. Phys. B388, 315 (1992).

[19] V. Bernard, N. Kaiser, and U.-G Meißner, Int. J. Mod. Phys. E 4, 193 (1995).

[20] V. Bernard, N. Kaiser, and U. G. Meißner, Nucl. Phys. A611, 429 (1996).

[21] H. Tang, hep-ph/9607436.

[22] P. J. Ellis and H. B. Tang, Phys. Rev. C 57, 3356 (1998).

[23] T. Becher and H. Leutwyler, Eur. Phys. J. C 9, 643 (1999).

[24] J. Gegelia and G. Japaridze, Phys. Rev. D 60, 114038 (1999).

[25] J. Gegelia, G. Japaridze, and X. Q. Wang, hep-ph/9910260.

[26] M. Lutz, Nucl. Phys. A677, 241 (2000).

[27] M. F. Lutz and E. E. Kolomeitsev, Nucl. Phys. A700, 193 (2002).

[28] P. J. Ellis and K. Torikoshi, Phys. Rev. C 61, 015205 (2000).

[29] B. Kubis and U.-G. Meißner, Nucl. Phys. A679, 698 (2001).

[30] S. L. Zhu, S. Puglia, and M. J. Ramsey-Musolf, Phys. Rev. D 63, 034002 (2001).

[31] B. Kubis and U.-G. Meißner, Eur. Phys. J. C 18, 747 (2001).

[32] S. L. Zhu, G. Sacco, and M. J. Ramsey-Musolf, Phys. Rev. D 66, 034021 (2002). 

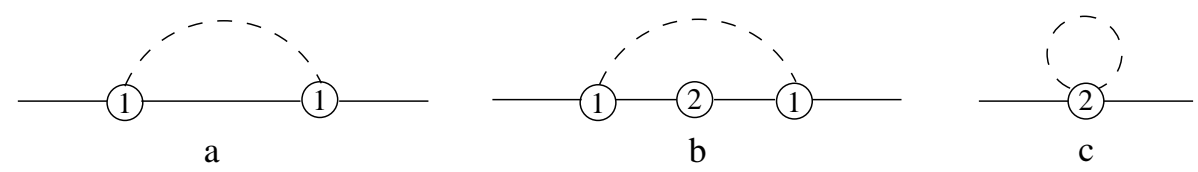

FIG. 1: One-loop contributions to the nucleon self-energy at $\mathcal{O}\left(q^{4}\right)$. The numbers in the interaction blobs denote the order of the Lagrangian from which they are obtained.

[33] T. Becher and H. Leutwyler, J. High Energy Phys. 06, 017 (2001).

[34] K. Torikoshi and P. J. Ellis, Phys. Rev. C 67, 015208 (2003).

[35] B. Borasoy and S. Wetzel, Phys. Rev. D 63, 074019 (2001).

[36] N. Beisert and B. Borasoy, Eur. Phys. J. A 11, 329 (2001).

[37] N. Beisert and B. Borasoy, Nucl. Phys. A705, 433 (2002).

[38] V. Bernard, T. R. Hemmert, and U.-G. Meißner, Phys. Lett. B 545, 105 (2002).

[39] V. Bernard, T. R. Hemmert, and U.-G. Meißner, Phys. Rev. D 67, 076008 (2003).

[40] J. Gasser, M. A. Ivanov, E. Lipartia, M. Mojžiš, and A. Rusetsky, Eur. Phys. J. C 26, 13 (2002).

[41] G. P. Lepage, in Proceedings of the 9th J. A. Swieca Summer School: Particles and Fields, Sao Paulo, Brazil, 1997, edited by J. C. A. Barata, A. P. C. Malbouisson, and S. F. Novaes (World Scientific, Singapore, 1998).

[42] D. B. Kaplan, M. J. Savage, and M. B. Wise, Phys. Lett. B 424, 390 (1998).

[43] J. Gegelia, in Proceedings of the Workshop on Methods of Nonperturbative Quantum Field Theory, Adelaide, Australia, 1998, edited by A. W. Schreiber, A. G. Williams, and A. W. Thomas (World Scientific, Singapore, 1998).

[44] H. W. Fearing and S. Scherer, Phys. Rev. D 53, 315 (1996).

[45] J. Bijnens, G. Colangelo, and G. Ecker, J. High Energy Phys. 02, 020 (1999).

[46] N. Fettes, U.-G. Meißner, M. Mojžiš, and S. Steininger, Ann. Phys. (N.Y.) 283, 273 (2000); 288, 249 (2001).

[47] T. Ebertshäuser, H. W. Fearing, and S. Scherer, Phys. Rev. D 65, 054033 (2002).

[48] J. Bijnens, L. Girlanda, and P. Talavera, Eur. Phys. J. C 23, 539 (2002).

[49] G. Colangelo, J. Gasser, and H. Leutwyler, Phys. Rev. Lett. 86, 5008 (2001).

[50] S. Weinberg, Nucl. Phys. B363, 3 (1991).

[51] W. Zimmermann, in Lectures on Elementary Particles and Quantum Field Theory, Brandeis University Summer Institute in Theoretical Physics, Volume 1, edited by S. Deser, M. Grisaru, and H. Pendleton (MIT Press, Cambridge, Massachusetts, 1970).

[52] G. Ecker, Prog. Part. Nucl. Phys. 35, 1 (1995).

[53] Handbook of Mathematical Functions, edited by M. Abramowitz and I. A. Stegun (Dover, New York, 1972).

[54] J. Gegelia, G. S. Japaridze and K. S. Turashvili, Theor. Math. Phys. 101, 1313 (1994).

[55] C. Itzykson and J. B. Zuber, Quantum Field Theory (McGraw-Hill, New York, 1980).

[56] H. Leutwyler, $\pi N$ Newslett. 15, 1 (1999).

[57] M. E. Sainio, $\pi N$ Newslett. 16, 138 (2002).

[58] T. Fuchs, M. Schindler, J. Gegelia, and S. Scherer, hep-ph/0308006, to appear in Phys. Lett. B. 